\title{
Es Posible Enfrentar el Cambio Climático y Producir más Leche y Carne con Sistemas Silvopastoriles Intensivos
}

\section{Enrique Murgueitio Restrepo ${ }^{1}$, Rolando Barahona Rosales ${ }^{2}$, Martha Xochilt Flores Estrada ${ }^{3}$, Julián David Chará Orozco ${ }^{4}$ y Julián Esteban Rivera Herrera ${ }^{5}$}

\begin{abstract}
Resumen. Reducir la vulnerabilidad a los desastres, adaptar los sistemas de producción agropecuaria a los cambios ambientales y mitigar las emisiones de gases con efecto de invernadero son acciones urgentes para enfrentar el cambio climático. En ganadería bovina de pastoreo, la investigación aplicada realizada en empresas y familias pioneras de varios países, ha permitido notables avances en agroforestería, especialmente con el sistema silvopastoril intensivo (SSPi), un uso de la tierra caracterizado por aplicar simultáneamente varios principios agroecológicos orientados a la maximización de la transformación de la energía solar en biomasa, la fijación biológica del nitrógeno, la solubilización del fósforo, la acumulación de materia orgánica en el suelo, el pastoreo rotacional, el uso inteligente del agua, la conservación de la diversidad biológica y el bienestar animal. Con estos arreglos, en los SSPi se aumenta la carga animal hasta cuatro o cinco veces en comparación al pastoreo extensivo pasando de 200 hasta $800-1200 \mathrm{~kg} \mathrm{ha}^{-1} \mathrm{año}^{-1}$, a la par que se reducen los costos en sistemas intensivos al reemplazar el alimento concentrado ( $40 \%$ en vacas lecheras tropicales) y evitar el uso de fertilizantes nitrogenados (bajan de $300 \mathrm{ha}^{-1}$ año ${ }^{-1}$ de $\mathrm{N}_{2}$ hasta cero). La producción de carne y leche por hectárea se incrementa ostensiblemente sin aumentos en las emisiones de metano entérico ni aumentos en las emisiones de óxido nitroso. De esta manera, la implementación de SSPi permite intensificar sosteniblemente la producción de carne y leche bovina en el trópico y subtrópico.
\end{abstract}

Palabras clave: Bienestar animal, ganadería tropical, gases efecto invernadero, sistemas agroforestales pecuarios.

\section{It is Possible to Address Climate Change and Produce More Milk and Meat with Intensive Silvopastoral Systems}

Abstract. Reducing vulnerability to disasters, adapting agricultural production systems to environmental changes, and mitigating emissions of greenhouse gases emissions are urgent actions to deal climate change. With regard to cattle grazing, applied research carried out in companies and pioneer families from several countries, has enabled remarkable progress in agro-forestry, especially with regards to intensive silvopastoral systems (ISS), a use of land characterized for applying simultaneously several agro ecological principles aimed at maximizing the conversion of solar energy into biomass, such as biological nitrogen fixation, phosphorus solubilization, accumulation of organic matter in the soil, rotational grazing, intelligent water usage, conservation of diversity biological, and animal welfare. With these arrangements, the SSPI allows stocking rate up four or five times greater compared to extensive grazing systems, going from 200 to $8001200 \mathrm{~kg} \mathrm{meat} \mathrm{ha}^{-1} \mathrm{yr}^{-1}$, while at the same time reducing costs in intensive grazing systems by reducing the use of feed concentrates $(40 \%$ in tropical dairy cows) and eliminating the need of nitrogen fertilizers (going from 300 to zero $\mathrm{N}_{2} \mathrm{~kg} \mathrm{ha}^{-1} \mathrm{yr}^{-1}$ ). The production of meat and milk per hectare is increased significantly and this increase is not accompanied by increases in enteric

\footnotetext{
${ }^{1}$ Director Ejecutivo Fundación Centro para la Investigación en Sistemas Sostenibles de Producción Agropecuaria - CIPAV, Colombia. Correo electrónico enriquem@fun.cipav.org.co

${ }^{2}$ Profesor Titular, Departamento de Producción Animal, Universidad Nacional de Colombia, Medellín. Colombia. Correo electrónico rbarahonar@unal.edu.co

${ }^{3}$ Fundación Produce Michoacán. Morelia, México. Correo electrónico dramxfe@fupromich.com

${ }^{4}$ Coordinación de Investigación, Fundación CIPAV, Colombia. Correo electrónico julian@fun.cipav.org.co

${ }^{5}$ Investigador en Ganadería Sostenible, Fundación CIPAV, Colombia. Correo electrónico jerivera@fun.cipav.org.co
} 
methane emissions or by increases in nitrous oxide emissions. Thus, the implementation of SSPI allows to sustainably intensify production of meat and milk cattle in the tropics and the subtropics.

Key words: Animal welfare, greenhouse gases, tropical cattle ranching, livestock agroforestry systems.

\section{Introducción}

Las gramíneas tropicales y subtropicales son la base de la alimentación ganadera en casi toda América Latina. Además de estar expuestas a un clima en constante transformación, son sometidas al pisoteo y pastoreo sin el uso de prácticas agronómicas como subsolado, fertilización, corrección de acidez, siembra de leguminosas y árboles y periodos de descansos adecuados. No en vano, los forrajes consumidos por los animales tienen calidad nutricional de media a baja (proteína, energía y minerales) lo cual está asociado al alto contenido de carbohidratos estructurales, bajo contenido de carbohidratos solubles, contenidos de proteína menor al 7\% y digestibilidad menor a 55\% (Barahona et al. 2014).

La baja carga animal producto de los aspectos anteriormente mencionados, conlleva a que en la mayor parte de los sistemas ganaderos de América Latina $y$ el Caribe registre bajos niveles de productividad y competitividad como consecuencia del agotamiento de los recursos naturales. A la par, en los últimos años los efectos negativos de la ganadería sobre el medio ambiente han recibido mucha publicidad negativa, generando que los gobiernos y ciudadanos presionen al sector ganadero por sus emisiones de gases con efecto de invernadero (GEI) como el dióxido de carbono $\left(\mathrm{CO}_{2}\right)$, metano $\left(\mathrm{CH}_{4}\right)$ y óxido nitroso $\left(\mathrm{N}_{2} \mathrm{O}\right)$ (Ku Vera et al. 2011).

Una de las soluciones más innovadoras a estos problemas son los sistemas silvopastoriles (SSP) con arreglos agroforestales en los que interactúan en forma simultánea plantas leñosas perennes (árboles o arbustos), plantas herbáceas o volubles (leguminosas herbáceas, pastos y arvenses) y animales domésticos, principalmente bovinos, équidos, ovinos y caprinos. Dentro de los SSP se destacan los sistemas silvopastoriles intensivos (SSPi), una modalidad de sistema agroforestal pecuario para la producción de carne, leche, madera, frutas y otros bienes asociados. En el SSPi interactúan en el mismo espacio y tiempo una o más especies de varios estratos. En el estrato herbáceo se encuentran gramíneas forrajeras nativas de América o introducidas, y plantas leguminosas herbáceas. El segundo estrato de vegetación tiene arbustos forrajeros como Leucaena leucocephala (Lam.) de Wit., de la subfamilia Mimosoidae; Tithonia diversifolia (Hemsl.) A. Gray, de la familia Asteracea; o Guazuma ulmifolia Lam., de la familia Malvaceae, en alta densidad (entre 10 y más de 40,000 plantas/ha ${ }^{1}$ ) para el ramoneo del ganado. El sistema incluye un tercer y hasta cuarto estrato o nivel trófico formado por árboles de todo tipo en la periferia y divisiones de los potreros, y árboles dispersos o en líneas, entre 25 y 200 árboles adultos/ha ${ }^{1}$ (Figura 1). El sistema incluye la oferta permanente de agua de buena calidad en bebederos móviles y sal mineralizada balanceada. En la periferia de los potreros se establecen cercas vivas y el ganado se maneja con cercas o cintas eléctricas fijas o móviles, según la tasa de rotación y la fisiología de las plantas forrajeras (Murgueitio et al. 2015).

\section{Aplicación de Principios Agroecológicos en los Sistemas Silvopastoriles Intensivos}

La intensificación ganadera con adaptación al cambio climático aplica principios agroecológicos para elevar la eficiencia de los procesos biofísicos esenciales, que maximizan la transformación de la energía solar en biomasa, la fijación biológica del nitrógeno, la solubilización del fósforo, la acumulación de materia orgánica en el suelo, el uso del pastoreo rotacional de alta carga instantánea con periodos de descanso largos, el uso de animales resistentes y adaptados al pastoreo-ramoneo en condiciones extremas de climas y alimentación, el uso inteligente del agua, el alto grado de bienestar de los animales, la conservación de la diversidad biológica, la captura de $\mathrm{CO}_{2}$, las menores emisiones de $\mathrm{CH}_{4}$, la elevada resiliencia al cambio climático y menor uso de agroquímicos, hormonas y antibióticos (Murgueitio et al. 2015). El cuadro 1 resume los principios agroecológicos más destacables que se aplican en los $\mathrm{SSPi}$, el componente principal que se beneficia y los autores de las publicaciones que lo respaldan. 


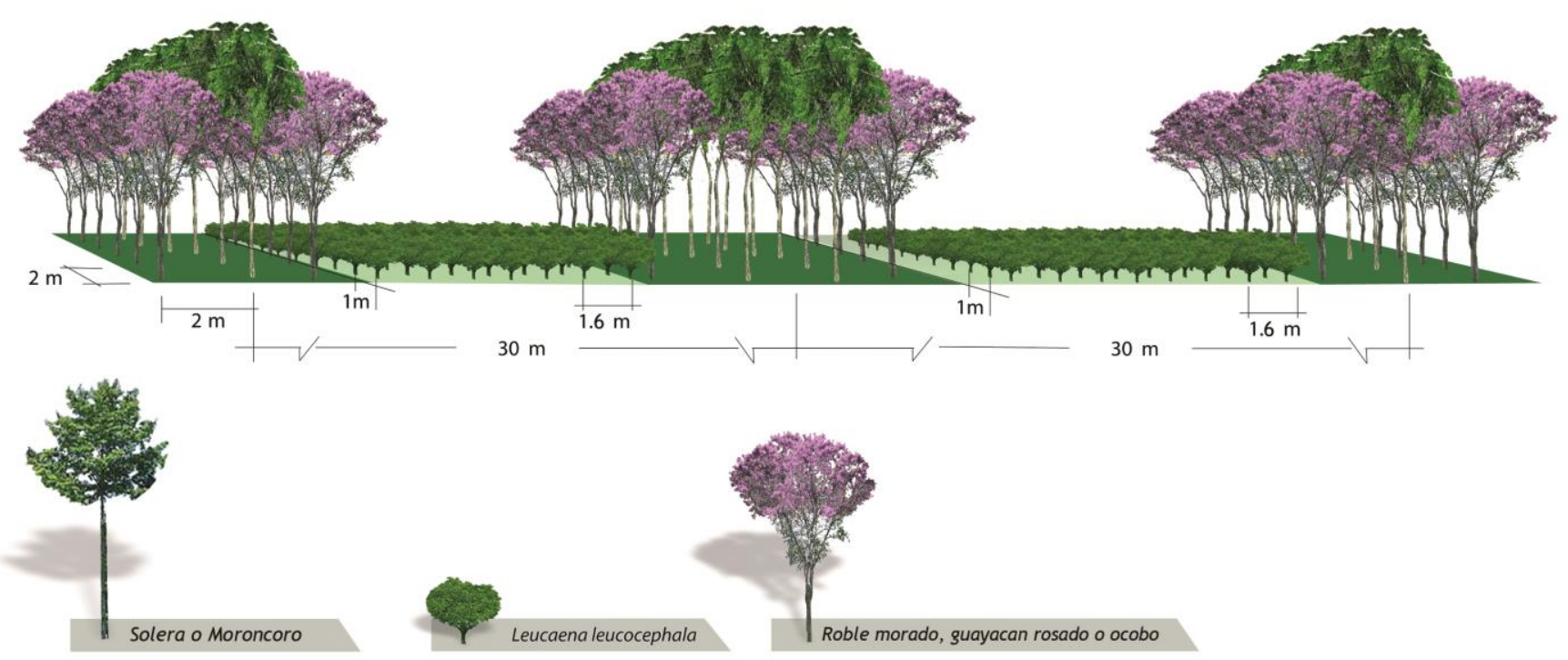

Figura 1. Esquema de un sistema silvopastoril intensivo con Leucaena leucocephala y pastos mejorados, asociados a una plantación de árboles maderables nativos (Calle y Murgueitio 2008).

\section{Es Rentable Producir Carne y Leche con los SSPi a Pesar del Cambio Climático}

Existen varios modelos tecnológicos que elevan la productividad ganadera de pastoreo, como aquellos que incluyen la fertilización y el riego, pero su implementación puede reducir la rentabilidad del sistema al aumentar los costos de producción. También está demostrado que la inversión en las divisiones de pastos para una rotación más eficiente, genera mayor productividad e ingresos con mejor competitividad por reducción en los costos de producción por unidad de leche y carne bovina (Holmann et al. 2003).

Debido a aumentos en la calidad nutricional de la dieta (Gaviria et al. 2015) y en consumo de nutrientes (Cuartas et al. 2015), en los SSPi se produce más carne y leche que en sistemas convencionales (Cuartas et al. 2014). Además, la ganadería basada en estos sistemas es más rentable (González 2013).

Los sistemas silvopastoriles intensivos son rentables en escenarios ganaderos de leche o carne porque se eleva hasta cuatro veces la producción pecuaria comparada con la de sistemas de pastoreo extensivo y también porque producen a menores costos que los sistemas intensivos con pastos fertilizados, regados y con oferta de alimentos concentrados. En los SSPi se aumenta la carga animal hasta cuatro o cinco veces frente al pastoreo extensivos, favoreciendo una mayor producción animal de carne al pasar de $200 \mathrm{~kg}^{-1} \mathrm{año}^{-1}$ a 800 o $1200 \mathrm{~kg}^{-1}$ año $^{-1}$ (Mahecha et al. 2011; Solorio-Sánchez et al. 2012). A su vez, la implementación de SSPi permite reducir los costos comparados con sistemas intensivos, al reemplazar el alimento concentrado en un $40 \%$ en vacas lecheras y reducir el uso de fertilizantes nitrogenados desde 300 ha $^{-1}$ año ${ }^{-1}$ o más hasta cero (Molina et al. 2011, Uribe et al. 2011). Finalmente, los SSPi se destacan por reducir la estacionalidad de la reproducción (Sierra et al. 2015) porque el ganado tiene mejor alimentación en épocas críticas de sequía y porque sufre menos estrés calórico con reducción entre 6 - 14 grados Celsius en la temperatura de su entorno (Murgueitio et al. 2013). Como consecuencia, la producción de carne y leche por hectárea se incrementa. 
Cuadro 1. Principios agroecológicos aplicados a los Sistemas Silvopastoriles Intensivos.

\begin{tabular}{|c|c|c|}
\hline Principio Agroecológico & $\begin{array}{l}\text { Componente principal } \\
\text { del SSPi involucrado }\end{array}$ & Referencias \\
\hline $\begin{array}{l}\text { Transformación máxima de } \\
\text { energía solar en biomasa vegetal }\end{array}$ & $\begin{array}{l}\text { Suelo, agua, pastos, } \\
\text { arbustos y árboles }\end{array}$ & $\begin{array}{l}\text { Arias-Giraldo 2009, Ayala-Burgos y Aguilar } \\
2011 \text {, Bacab-Pérez y Solorio } 2011 \text { y Gaviria et } \\
\text { al. } 2015\end{array}$ \\
\hline Fijación biológica de nitrógeno & $\begin{array}{l}\text { Suelo, estrato arbustivo } \\
\text { de } L \text {. leucocephala }\end{array}$ & $\begin{array}{l}\text { Barahona et al. 2014, Nair et al. } 2007 \text { y } \\
\text { Solorio-Sánchez et al. } 2012\end{array}$ \\
\hline $\begin{array}{l}\text { Solubilización del fósforo en } \\
\text { suelos ácidos }\end{array}$ & $\begin{array}{l}\text { Suelo, estrato arbustivo } \\
\text { de } T \text {. diversifolia }\end{array}$ & $\begin{array}{l}\text { Mauricio et al. 2014, Nziguheba et al. } 2002 \text { y } \\
\text { Pérez et al. } 2009\end{array}$ \\
\hline $\begin{array}{l}\text { Acumulación de materia orgánica } \\
\text { en los suelo }\end{array}$ & $\begin{array}{l}\text { Suelo, estratos } \\
\text { arbustivo y arbóreo; } \\
\text { ganado }\end{array}$ & $\begin{array}{l}\text { Chará et al. 2015, Montagnini et al. } 2013 \text { y } \\
\text { Nair et al. } 2007\end{array}$ \\
\hline Mayor reciclaje de nutrientes & $\begin{array}{l}\text { Suelo, pastos, arbustos, } \\
\text { árboles. }\end{array}$ & $\begin{array}{l}\text { Calle et al. 2014, Casanova et al. } 2010 \text { y } \\
\text { Vallejo et al. } 2010\end{array}$ \\
\hline $\begin{array}{l}\text { Pastoreo rotacional de alta carga } \\
\text { animal instantánea con largos } \\
\text { periodos de descanso }\end{array}$ & $\begin{array}{l}\text { Estratos de pastos y } \\
\text { arbustivo; ganado }\end{array}$ & $\begin{array}{l}\text { Galindo et al. 2010, Murgueitio et al. 2011, } \\
\text { Murgueitio et al. } 2014 \text { y Uribe et al. } 2011\end{array}$ \\
\hline $\begin{array}{l}\text { Empleo de animales resistentes y } \\
\text { adaptados al pastoreo - ramoneo } \\
\text { en climas y alimentación } \\
\text { limitantes }\end{array}$ & Ganado & Molina C. et al. 2011 y Murgueitio et al. 2013 \\
\hline $\begin{array}{l}\text { Uso eficiente del agua para oferta } \\
\text { del ganado siempre y con buena } \\
\text { calidad }\end{array}$ & Ganado & $\begin{array}{l}\text { Montoya et al. 2015, Murgueitio et al.. } 2015 \text { y } \\
\text { Uribe et al. } 2011\end{array}$ \\
\hline Alto grado de bienestar animal & $\begin{array}{l}\text { Ganado, estrato de } \\
\text { árboles }\end{array}$ & $\begin{array}{l}\text { Broom et al. 2013, Reyes } 2015 \text { y Tarazona et } \\
\text { al. } 2013\end{array}$ \\
\hline Conservación de la biodiversidad & $\begin{array}{l}\text { Suelo, agua, estratos } \\
\text { de pastos, arbustos y } \\
\text { árboles }\end{array}$ & $\begin{array}{l}\text { Calle et al. 2014, Fajardo et al. 2010, Giraldo } \\
\text { et al. } 2010 \text { y Rivera L. et al. } 2013\end{array}$ \\
\hline Elevada Captura de $\mathrm{CO}_{2}$ & $\begin{array}{l}\text { Suelo, pasturas, } \\
\text { estratos arbustivos y de } \\
\text { árboles }\end{array}$ & $\begin{array}{l}\text { Chará et al. 2015, Harrison et al. 2015, } \\
\text { lbrahim et al. 2010, Naranjo et al. } 2012 \text { y } \\
\text { Montagnini et al. } 2013\end{array}$ \\
\hline Menores emisiones de $\mathrm{CH}_{4}$ y $\mathrm{N}_{2} \mathrm{O}$ & $\begin{array}{l}\text { Ganado, suelo, } \\
\text { excretas animales }\end{array}$ & $\begin{array}{l}\text { Ku-Vera et al. 2011, Harrison et al. 2015, } \\
\text { Molina I. et al. 2015, Molina I. et al. 2016, } \\
\text { Naranjo et al. 2012, Rivera J. et al. 2015a, } \\
\text { Rivera J. et al. 2015b y Cuartas et al. } 2014\end{array}$ \\
\hline $\begin{array}{l}\text { Alta capacidad de resiliencia al } \\
\text { cambio climático }\end{array}$ & $\begin{array}{l}\text { Suelo, agua, estratos } \\
\text { de pastos, arbustos y } \\
\text { árboles; ganado }\end{array}$ & $\begin{array}{l}\text { Harvey et al. 2013, Ibrahim et al. } 2010 \text { y } \\
\text { Molina et al. } 2011\end{array}$ \\
\hline $\begin{array}{l}\text { Producción de carne y leche con } \\
\text { mínimo empleo de agroquímicos, } \\
\text { antibióticos y hormonas }\end{array}$ & $\begin{array}{l}\text { Suelo, agua, pasturas y } \\
\text { ganado }\end{array}$ & $\begin{array}{l}\text { Corral-Flores et al. 2012, Barahona et al. } \\
\text { 2014, Mahecha et al. 2011, Paciullo et al. } \\
2014 \text { y Solorio-Sánchez et al. } 2012\end{array}$ \\
\hline
\end{tabular}

Los estudios financieros de engorde de ganado en el Caribe seco de Colombia muestran las ventajas del SSPi frente al pastoreo rotacional sin fertilizantes. Indicadores financieros como el ingreso bruto $/ \mathrm{ha}^{-1}$, la utilidad $/ \mathrm{ha}^{-1} / \mathrm{año}^{-1}$ y la tasa interna de retorno son mayores para SSPi, ya que son sistemas muy rentables al permitir ingresos brutos de US \$2,935 haaño ${ }^{-1}$ solo con pastos y arbustos y de US $\$ 3,839 \mathrm{ha}^{-1}$ año ${ }^{-1}$ cuando se incluye el negocio forestal (madera de eucalipto) en la misma área, lo que significa contar con 
Murgueitio et al.: Es Posible Enfrentar el Cambio Climático y Producir más Leche y Carne con Sistemas

US $\$ 669 / \mathrm{ha}^{-1} / \mathrm{año} \mathrm{O}^{-1}$. La tasa interna de retorno (TIR) con interés anual del $10 \%$, fue alta comparada con otras opciones (32.7\%) y se incrementa en $4.3 \%$ cuando hay venta de madera (Murgueitio et al. 2015). En el Pacífico de México (Michoacán) para los SSPi con riego por gravedad, la TIR alcanzó 33.5\%, comparado con alternativas convencionales ganaderas con TIR entre 12 y $18 \%$, y el periodo de recuperación de la inversión es en 4 años y con tasas de interés del $12 \%$ anual se da en 5 años (González-Pérez y SolorioSánchez 2012). Ambas regiones están sometidas a oscilaciones climáticas grandes, especialmente por sequías, reducción en los tiempos de lluvias y altas temperaturas ambientales debido al cambio climático.

Estudios en sistemas de doble propósito y lechería tropical en Colombia coinciden en demostrar la rentabilidad de los SSPi y dejan claro que los períodos necesarios para liberar la inversión están entre 3 y 4 años según la línea de base y el tamaño del predio (Reyes 2015).

\section{Emisiones de Metano $\left(\mathrm{CH}_{4}\right)$ y otros GEI}

En varios estudios se evidencia la capacidad de los SSPi basados en $L$. leucocephala para reducir las emisiones de GEI en sistemas bovinos orientados a la producción de carne y leche. Estas determinaciones incluyeron la producción de $\mathrm{CH}_{4}$ por fermentación entérica in vivo (Molina et al. 2015; Molina et al. 2016) $y$ in vitro (Rivera et al. 2015a), determinación de huellas de carbono, medición de los flujos de gases provenientes de praderas y excreciones bovinas, así como el balance de GEI (Naranjo et al. 2012, Rivera et al. 2015a, Rivera 2015b).

En lo referente a las emisiones entéricas de $\mathrm{CH}_{4}$, Molina et al. (2016) encontraron que con una inclusión de $L$. leucocephala de un $25 \%$ en dietas basadas en C. plectostachyus ofrecidas a novillas, estas emisiones pueden disminuir en $15 \%$ por kilogramo de materia seca consumida (MSC).

\section{Conclusión}

Es posible enfrentar el cambio climático en sistemas de pastoreo para producir carne y leche en el trópico y subtrópico en forma rentable y sostenible mediante la adopción de sistemas silvopastoriles intensivos que se soportan en principios agroecológicos investigados y documentados en América Latina y el Caribe.

\section{Literatura Citada}

Arias-Giraldo, L.M, J.C. Camargo, M.A. Dossman, M.A. Echeverry, J.A. Rodríguez, C.H. Molina, E.J. Molina e I.D. Melo. 2009. Estimación de biomasa aérea y desarrollo de modelos alométricos para Leucaena leucocephala en sistemas silvopastoriles de alta densidad en el valle del Cauca, Colombia. Recursos Naturales y Ambiente 58:32-39.

Ayala-Burgos, A. y C. Aguilar-Pérez. 2011. Balance energético/proteico para intensificar la producción animal en los sistemas silvopastoriles. III Congreso sobre Sistemas Silvopastoriles Intensivos para la ganadería sostenible del siglo XXI. Morelia, Michoacán, México.

Bacab-Pérez, H.M. y F.J. Solorio-Sánchez. 2011. Oferta y consumo de forraje y producción de leche en ganado de doble propósito manejado en sistemas silvopastoriles en Tepalcatepec, Michoacán. Tropical and Subtropical. Agroecosystems 13:271-278.

Barahona, R., M.S. Sánchez, E. Murgueitio y J.D. Chará. 2014. Contribución de la Leucaena leucocephala Lam (de Wit) a la oferta y digestibilidad de nutrientes y las emisiones de metano entérico en bovinos pastoreando en sistemas silvopastoriles intensivos. En: Premio Nacional de Ganadería José Raimundo Sojo Zambrano, modalidad Investigación Científica. Bogotá, Colombia, Revista Carta Fedegán 140:66-69.

Broom, D.M., F.M. Galindo y E. Murgueitio. 2013. Sustainable, efficient livestock production with high biodiversity and good welfare for animals. Proceedings of the Royal Society Biological Sciences 280:20132025.

Calle, Z., J.D. Chará, E. Murgueitio y C. Giraldo. 2014. Intensive silvopastoral systems: integration of sustainable cattle ranching. In: Calle, A., Calle, Z., Garen, E., Del Cid-Liccardi, A. (eds.), Ecological Restoration and Sustainable Agricultural Landscapes. Environmental Leadership and Training Initiative, New Haven, CT. Disponible en: http://www.fao.org/fileadmin/user upload/nr/sustainabilit y pathways/docs/Colombia Murgueitio Mixed Species Silvopastoral systems.pdf

Calle, Z., Murgueitio, E. 2008. El roble morado o guayacán rosado Tabebuia rosea (Bertol.) D.C.: explosión de belleza en los paisajes ganaderos. Revista Carta FEDEGAN 109: 76 - 82

Casanova, L. F., M.J. Caamal, A.J. Petit, S.F. Solorio y C.J. Castillo. 2010 Acumulación de carbono en la biomasa de Leucaena leucocephala y Guazuma ulmifolia asociadas $y$ en monocultivo. Revista Forestal Venezolana 54 (1): 45-50.

Corral-Flores, G., M.E. Rodríguez-Echavarría, B. SolorioSánchez, A.D. Alarcón-Rojo, J.A. Grado-Ahuir, C. Rodríguez-Muela, L. Cortés-Palacios, V.E. Segovia- 
Beltrán y F.J. Solorio-Sánchez. 2012. Calidad de la carne de bovinos engordados en un sistema silvopastoril intensivo en dos épocas del año. En: Memorias, IV Congreso Internacional Sobre Sistemas Silvopastoriles Intensivos en la Ganadería con Ciencia [Morelia, México, 21-23 mar. 2012]. p. 113-122.

Cuartas Cardona, C.A., J.F. Naranjo Ramírez, A. Tarazona Morales, E. Murgueitio Restrepo, J.D. Chará Orozco, J. Ku Vera y R. Barahona Rosales. 2014. Contribution of intensive silvopastoral systems to animal performance and to adaptation and mitigation of climate change. Revista Colombiana de Ciencias Pecuarias 27(2): 7694.

Cuartas, C.A., J.F. Naranjo, A. Tarazona, G. Correa y R. Barahona. 2015. Dry matter and nutrient intake and diet composition in Leucaena leucocephala - based intensive silvopastoral systems. Tropical and Subtropical Agroecosystems 18: $303-311$.

Chará, J., J.C. Camargo, Z Calle, L. Bueno, E. Murgueitio, L. Arias, M. Dossman y C.H. Molina. 2015. Servicios ambientales de Sistemas Silvopastoriles Intensivos: mejora en propiedades del suelo y restauración ecológica. En: Montagnini, F., Somarriba, E., Murgueitio, E., Fassola, H., Eibl, B. (Eds.). Sistemas Agroforestales. Funciones productivas, socioeconómicas y ambientales. Serie Técnica Informe Técnico 402, CATIE, Turrialba, Costa Rica. Fundación CIPAV. Cali, Colombia. 454pp.

Fajardo, D., R. Johnston, L. Neira, J.D. Chará y E. Murgueitio. 2010. Influencia de los sistemas silvopastoriles en la diversidad de aves en la cuenca del río La Vieja, Colombia. Recursos Naturales y Ambiente 58:9-16.

Galindo, W., J.F. Naranjo, M.M. Murgueitio, V.A. Galindo y R. Tatis, R. 2010. Producción de carne bovina con sistemas silvopastoriles intensivos basados en Guazuma ulmifolia y otras especies en la región del Caribe seco de Colombia (en línea) In: Ibrahim, M, Murgueitio, E. (Eds.). Actas, VI Congreso Latinoamericano Agroforestería para la Producción Agropecuaria Sostenible [Panamá, Panamá, 28-30 sept. 2010]. En: http://www.cipav.org.co/red de agro/Panama2010.html

Gaviria, X., J.E Rivera y R. Barahona. 2015. Nutritional quality and fractionation of carbohydrates and protein in the forage components of an intensive silvopastoral system. Pastos y Forrajes, 38, (2): 194-201.

Giraldo, C., F. Escobar, J.D. Chará y Z. Calle. 2010. The adoption of silvopastoral systems promotes recovery of ecological processes regulated by dung beetles in the Colombian Andes. Insect Conservation and Diversity $4(2): 115-122$.
González, J.M. 2013. Costos y beneficios de un sistema silvopastoril intensivo (SSPI), con base en Leucaena leucocephala. Estudio de caso en el municipio de Tepalcatepec, Michoacán, México. Avances en Investigación Agropecuaria. 17(3): 35-50

González-Pérez, J.M. y B. Solorio-Sánchez. 2012. Indicadores sociales y económicos de los SSPI del valle de Tepalcatepec, Michoacán, México, cinco años de madurez. p. 209-217 En: Memorias, IV Congreso Internacional sobre Sistemas Silvopastoriles Intensivos. Morelia, Michoacán, México, Fundación Produce Michoacán, Universidad Autónoma de Yucatán.

Harrison, M., C. McSweeney, N.W. Tomkins y R.J. Eckard. 2015. Improving greenhouse gas emissions intensities of subtropical and tropical beef farming systems using Leucaena leucocephala. Agricultural Systems 136, 138146.

Harvey, C., M. Chacón, C. Donatti, E. Garen, L. Hannah, L. A. Andrade, L. Bede, D. Brown, A. Calle, J.D. Chará, C Clement, E. Gray, M. Hoang, P. Minang, A. Rodríguez, C. Seeberg-Elverfeldt, B Semroc, S. Shames, S. Smukler, E. Somarriba, E. Torquebiau, J. van Etten y E. Wollenberg. 2013. Climate-smart Landscapes: Opportunities and Challenges for Integrating Adaptation and Mitigation in Tropical Agriculture. Conservation Letters 7(2):77-90.

Holmann, F., L. Rivas, J. Carulla, B. Rivera, L.A. Giraldo, S. Guzmán, M. Martínez, A. Medina y A. Farrow. 2003. Evolution of Milk Production Systems in Tropical Latin America and its interrelationship with Markets: An Analysis of the Colombian Case. Livestock Research for Rural Development 15(9). En: http://www.Irrd.org/lrrd15/9/holm159.htm

Ibrahim, M., L. Guerra, F. Casasola y C. Neely. 2010. Importance of silvopastoral systems for mitigation of climate change and harnessing of environmental benefits. In FAO, edited by M. Abberton, R. Conant, C. Batello, Grassland carbon sequestration: management, policy and economics. Proceedings of the workshop on the role of grassland carbon sequestration in the mitigation of climate change. Integrated Crop Management, Vol. 11. Rome, FAO.

Ku Vera, J.C., G.A. Ruiz, M.S. Albores, P.E. Briceño, H.C. Espinoza, R.N. Ruiz, L.M. Contreras, A.J. Ayala y L. Ramírez. 2011. Alimentación de rumiantes en Sistemas Silvopastoriles Intensivos: Avances de investigación básica. Memorias, $3^{\circ}$ Congreso sobre Sistemas Silvopastoriles Intensivos para la ganadería sostenible del siglo XXI. [Morelia, Michoacán, México, 2-4 mar. 2011]. p. 8-16. 
Mahecha, L., M.M. Murgueitio, J. Angulo, M. Olivera, A. Zapata, C.A. Cuartas, J.F. Naranjo y E. Murgueitio. 2011. Desempeño animal y características de la canal de dos grupos raciales de bovinos doble propósito pastoreando en sistemas silvopastoriles intensivos. Revista Colombiana de Ciencias Pecuarias 24(3):470.

Mauricio, R.M., R.S. Ribeiro, S.R. Silveira, P.L Silva, L. Calsavara, L.G.R. Pereira y D.S. Paciullo. 2014. Tithonia diversifolia for ruminant nutrition. Tropical Grasslands - Forrajes Tropicales. 2, 82-84.

Molina, C.H., E.J. Molina, C. Giraldo, Z. Calle y E. Murgueitio. 2011. Resiliencia de los sistemas silvopastoriles intensivos a los efectos de cambio climático en el Valle del Cauca, Colombia. Memorias, $3^{\circ}$ Congreso sobre Sistemas Silvopastoriles Intensivos para la ganadería sostenible del siglo XXI. [Morelia, Michoacán, México, 2-4 mar. 2011]. p. 208-214.

Molina, I.C., E. A. Angarita, O.L. Mayorga, J.D. Chará, J. y R. Barahona. 2016. Effect of Leucaena leucocephala on methane production of Lucerna heifers fed a diet based on Cynodon plectostachyus. Livestock Science185: 2429

Molina, I.C., G. Donney's, S. Montoya, J.E. Rivera, G. Villegas, J.D. Chará y R. Barahona. 2015. The inclusion of Leucaena leucocephala reduces the methane production in Lucerne heifers receiving a Cynodon plectostachyus and Megathyrsus maximus diet. Livestock. Research for Rural. Development. 27, 96 〈http://www.Irrd.org .

Montagnini, F., I. Ibrahim y E. Murgueitio. 2013. Silvopastoral systems and climate change mitigation in Latin America. Bois et Forêts des Tropiques 316(2):316.

Montoya, S., G. Villegas, I. Molina, G. Doneys, J.D. Chará y R. Barahona. 2015. Comparación del consumo de forraje y agua y sus correlaciones en un sistema silvopastoril intensivo y uno convencional en tres regiones de Colombia. En: Memorias $3^{\circ}$ Congreso Nacional de Sistemas Silvopastoriles y VIII Congreso Internacional de Sistemas Agroforestales. Puerto Iguazú, Misiones, Argentina. INTA. 117 - 122 pp.

Murgueitio, E., J.D. Chará, R. Barahona, C. A. Cuartas y J.F. Naranjo. 2014. Intensive Silvopastoral Systems (ISPS), mitigation and adaptation tool to climate change. Tropical and Subtropical Agroecosystems 17(3). 501507.

Murgueitio, E., Z. Calle, F. Uribe, A. Calle y B. Solorio. 2011. Native trees and shrubs for the productive rehabilitation of tropical cattle ranching lands. Forest Ecology and Management 261:1654-1663.

Murgueitio, E., J.D. Chará, A. Solarte, F. Uribe, C. Zapata y J.E. Rivera. 2013. Agroforestería Pecuaria y Sistemas Silvopastoriles Intensivos (SSPi) para la adaptación ganadera al cambio climático con sostenibilidad. Universidad de Antioquia, Colombia, Revista Colombiana de Ciencias Pecuarias (RCCP) 26:313-316.
Murgueitio, E., M.X. Flores, Z. Calle, J.D. Chará, R. Barahona, C.H. Molina y F. Uribe. 2015. Productividad en sistemas silvopastoriles intensivos en América Latina. p. 59-101. En: Montagnini, F., Somarriba, E., Murgueitio, E., Fassola, H., Eibl, B. (eds.). Sistemas Agroforestales. Funciones productivas, socioeconómicas y ambientales. Serie Técnica Informe Técnico 402, CATIE, Turrialba, Costa Rica. Fundación CIPAV. Cali, Colombia. 454 p.

Nair, V.D., S.G. Haile, G.A. Michel y R. Nair. 2007. Environmental quality improvement of agricultural lands through silvopasture in southeastern United States. Scientia Agricola 64(5): 513-519.

Naranjo, J.F., C. A. Cuartas, E. Murgueitio, J.D. Chará y R. Barahona. 2012. Balance de gases de efecto invernadero en sistemas silvopastoriles intensivos con Leucaena leucocephala en Colombia (en línea). Livestock Research for Rural Development 24(8). Disponible en: http://www.Irrd.org//rrd24/8/nara24150.htm

Nziguheba, G., R. Merckx, C.A. Palm y P. Mutuo. 2002. Combining Tithonia diversifolia and fertilizers for maize production in a phosphorus deficient soil in Kenya. Agroforestry Systems 55: 165-174.

Paciullo, D. S. C., M.F.A. Pires, L.J.M. Aroeira, M.J.F. Morenz, R.M. Maurício, C.A.M. Gomide y S.R. Silveira. 2014. Sward characteristics and performance of dairy cows in organic grass-legume pastures shaded by tropical tres. Animal 8 (8):1264-1271

Pérez, A., I. Montejo, J. Iglesias, O. López, G. Martín, D. García, Y. Milián y A. Hernández. 2009. Tithonia diversifolia (Helms.) A. Gray (en línea). Revista Pastos y Forrajes 32(1):1-15.

Reyes, E. 2015. Análisis de los beneficios de la adopción de sistemas silvopastoriles en la Producción de carne y leche en Colombia. (Estudios de caso). En: $3^{\mathrm{er}}$ Congreso Nacional de Sistemas Silvopastoriles: VII Congreso Internacional Sistemas Agroforestales / Compilado por Pablo L. Peri. - $1^{\underline{a}}$ ed. Santa Cruz. Ediciones INTA Argentina, 2015. p 459 - 462.

Rivera, J.E., I.C. Molina, G. Donney's, G. Villegas, J.D. Chará y R. Barahona, R. 2015a. Dinámica de fermentación y producción de metano en dietas de sistemas silvopastoriles intensivos con Leucaena leucocephala y sistemas convencionales orientados a la producción de leche. Livestock Research for Rural. Development. 27 〈http://www.Irrd.org//rrd27/4/rive270680.html〉.

Rivera, J., J.D. Chará, E. Murgueitio y R. Barahona. 2015b. Estimación de la huella de carbono en sistemas silvopastoriles intensivos y convencionales para la producción de leche bovina en Colombia. En: Memorias $3^{\circ}$ Congreso Nacional de Sistemas Silvopastoriles y VIII Congreso Internacional de Sistemas Agroforestales. Puerto Iguazú, Misiones, Argentina. INTA. 606 - 610 p. 
Rivera, L., I. Armbrecht, and Z. Calle. 2013. Silvopastoral systems and ant diversity conservation in a cattledominated landscape of the Colombian Andes. Agriculture, Ecosystems and Environment 181: 188194.

Sierra, E., T. Ruiz, J., Chará, R. Barahona, y J. F. Suárez. 2015. Conducta reproductiva de vacas lecheras cruzadas pastoreando en sistemas silvopastoriles intensivos del bosque seco tropical colombiano. . En: Memorias $3^{\circ}$ Congreso Nacional de Sistemas Silvopastoriles y VIII Congreso Internacional de Sistemas Agroforestales. Puerto Iguazú, Misiones, Argentina. INTA. 89 - 92 p.

Solorio-Sánchez, F.J., B. Solorio-Sánchez, F. CasanovaLugo, L. Ramírez-Avilés, A. Ayala-Burgos, J. Ku-Vera y C. Aguilar-Pérez. 2012. Situación actual global de la investigación y desarrollo tecnológico en el establecimiento, manejo y aprovechamiento de los sistemas silvopastoriles intensivos. In F.J. SolorioSánchez, C. Sánchez-Brito y J. Ku-Vera, (eds.), Memorias IV Congreso Internacional sobre Sistemas Silvopastoriles Intensivos. Morelia, México, Fundación Produce Michoacán, Universidad Autónoma de Yucatán, México.
Tarazona, A.M., M.C. Ceballos, C.A. Cuartas J.F. Naranjo, E. Murgueitio y R. Barahona. 2013. The relationship between nutritional status and bovine welfare associated to adoption of intensive silvopastoral systems in tropical conditions. In: Enhancing animal welfare and farmer income through strategic animal feeding - Some case studies, Edited by Harinder P.S. Makkar pages pp 69 - 78; FAO Animal Production and Health Paper No. 175. Rome, Italy. www.fao.org/docrep/017/i3164e/i3164e00.pdf.

Uribe, F., A.F. Zuluaga, L. Valencia, E. Murgueitio, A. Zapata y L. Solarte. 2011. Establecimiento y manejo de sistemas silvopastoriles. Manual 1. Proyecto Ganadería Colombiana Sostenible (en línea). Bogotá, Colombia, GEF, The World Bank, FEDEGAN, CIPAV, Fondo Acción, TNC. 78 p. Disponible En: http://www.cipav.org.co/pdf/1.Establecimiento.y.manejo. de.SSP.pdf

Vallejo, V. E., F. Roldán y R.P. Dick. 2010. Soil enzymatic activities and microbial biomass in an integrated agroforestry chronosequence compared to monoculture and a native forest of Colombia. Biology and Fertility of Soils 46 (6): 577-587.

Recibido para publicación el 21 de marzo de 2016. Aceptado para publicación el 16 de julio de 2016. 